\title{
A SET OF FOUR POSTULATES FOR BOOLEAN ALGEBRA IN TERMS OF THE "IMPLICATIVE" OPERATION*
}

\author{
BY \\ B. A. BERNSTEIN
}

1. Introduction. Whitehead and Russell's Principia Mathematica makes fundamental the notion " $\supset$ " of "implication," defined by

$$
p \supset q \cdot=\cdot \sim p \vee q, \text { Df. }
$$

The main object of my paper is to present in terms of this "implicative" operation $\dagger>$ a set of four postulates for Boolean algebra. This will secure for Boolean algebra, for the first time, a set of postulates expressed in terms of an operation other than "rejection" having as few postulates as the present minimum sets. $\ddagger$ Of course, by the principle of duality in Boolean algebra, my postulates will also be a set in terms of the dual of $p \supset q$, namely $\sim p q$.

I prove for my postulates (a) their consistency, (b) their mutual independence, (c) their sufficiency for Boolean algebra, (d) their necessariness for Boolean algebra. $\S$ The consistency and independence systems are all Boolean

* Presented to the Society, June 19, 1933; received by the editors October 9, 1933.

$\dagger$ The Principia calls $\supset$ a relation.

$\ddagger$ For the present minimum sets, see B. A. Bernstein, (I) A set of four independent postulates for Boolean algebras, these Transactions, vol. 17 (1916), pp. 50-51; (II) Simplification of the set of four postulates for Boolean algebras in terms of rejection, Bulletin of the American Mathematical Society, vol. 39 (1933), pp. 783-787. For another set of postulates in terms of $\supset$, the first set in terms of $\supset$, see E. V. Huntington, (I) A new set of independent postulates for the algebra of logic, with special reference to Whitehead and Russell's Principia Mathematica, Proceedings of the National Academy of Sciences, vol. 18 (1932), pp. 179-180. Huntington's postulates are eight in number (including an inadvertently omitted existence postulate).

$\S$ I offer (a)-(d) as a set of defining properties of a set of postulates: a system of propositions $S$ is a set of postulates for a (consistent) system $\Sigma$ if and only if the propositions of $S$ are (a) consistent, (b) mutually independent, (c) sufficient for $\Sigma$, (d) necessary for $\Sigma$. More simply and less formally stated, a set of postulates for a system is a set of propositions of the system which cannot be derived from one another but from which all the other propositions of the system can be derived. This view of postulates is opposed to the view, seemingly held widely, that demands of postulates only sufficiency and necessariness (hence also consistency). The latter view would have to accept as a set of postulates for euclidean geometry all of Euclid's Elements, and would violate the generally accepted distinction between postulate and theorem. My view of postulates is of course opposed to the view, seemingly held by some, that demands of postulates only sufficiency. This view would have to accept $S$ as a set of postulates for $\Sigma$, not only when $S$ is the whole of $\Sigma$, but also when $S$ is inconsistent (since "a false proposition implies any proposition") and when $S$ is only a special case of $\Sigma$ (when $S$, for example, is the theory of Abelian groups and $\Sigma$ the theory of groups in general). If $I$ am correct in my view, the term "independent postulates," found in the literature, must be understood to mean "postulates whose independence has been proved," and the term "postulates" applied to $S$ when only (c) and (d) have 
and very simple. The proof of sufficiency consists in deriving from the postulates my second set of postulates in terms of rejection (see my Paper II, loc. cit.); the proof of necessariness consists in the converse derivation.

I shall derive from my postulates the "theory of deduction" of the Principia. This will verify the fact, obtained elsewhere* less directly and from another point of view, that the theory of deduction is derivable from the general logic of classes.

There is a close relation between $\supset$ and the operation " - " of "exception" used by mef, and later by Taylor $\ddagger$, in postulates for Boolean algebra. I shall bring out this relation.

If in a set of independent postulates for the logic of classes there is a proposition demanding that the number of elements be at least two, and if this proposition be replaced by a proposition demanding that the number of elements be just two, then the propositions resulting from the change will be sufficient for the logic of propositions as a two-element Boolean algebra. But these propositions will, in general, not be independent. I have so chosen my postulates that the change in question will render them a set of independent postulates for the logic of propositions. $\S$

2. The postulates. The postulates with which we are mainly concerned have as primitive ideas a class $K$ and a binary operation $\supset$, and are the propositions $A_{1}-A_{4}$ below. $\|$ In Postulates $A_{2}$ and $A_{3}$, there is to be understood the supposition that the elements involved and their indicated combinations belong to $K$. This must especially be borne in mind when the independence of the postulates is considered. The postulates follow.

Postulate $A_{1} . a \supset b$ is an element of $K$ whenever $a$ and $b$ are elements of $K$.

been proved for $S$, must be understood to mean "provisional postulates" for $\Sigma$. If desired, "provisional postulates" might have a distinctive name, say basic propositions of $\Sigma$, or defining conditions for $\Sigma$.

* B. A. Bernstein, (III) On section A of Principia Mathematica, Bulletin of the American Mathematical Society, vol. 39 (1933), pp. 788-792.

† B. A. Bernstein, (IV) A complete set of postulates for the logic of classes expressed in terms of the operation "exception," and a proof of the independence of a set of postulates due to Del Re, University of California Publications in Mathematics, vol. 1, pp. 87-96.

$\ddagger$ J. S. Taylor, A set of five postulates for Boolean algebras in terms of the operation "exception," University of California Publications in Mathematics, vol. 1, pp. 242-248.

$\S$ For a discussion of the nature of the logic of propositions, see B. A. Bernstein, (V) Sets of postulates for the logic of propositions, these Transactions, vol. 28 (1926), pp. 472-478.

|| The symbol " =" used in the postulates is taken as an idea outside the system. Compare my (VI) Whitehead and Russell's theory of deduction as a mathematical science, Bulletin of the American Mathematical Society, vol. 37 (1931), pp. 480-488. For sets of postulates for Boolean algebra in which " $="$ is taken as an idea within the system, see E. V. Huntington, (II) New sets of independent postulates for the algebra of logic, with special reference to Whitehead and Russell's Principia Mathematica, these Transactions. vol. 35 (1933), pp. 274-304. 
Postulate $\mathrm{A}_{2} .(a \supset b) \supset a=a$.

Postulate A. $_{\mathbf{3}}$. There is an element $z$ in $K$ such that

$$
(d \supset d) \supset[(a \supset b) \supset c]=\{[(c \supset z) \supset a] \supset[(b \supset c) \supset z)]\}>z .
$$

Postulate $\mathrm{A}_{4} . K$ consists of at least two distinct elements.

3. Consistency and independence of the postulates. The consistency and the independence of Postulates $A_{1}-A_{4}$ are given by the following systems $S_{0}-S_{4}$, in which $S_{0}$ is the consistency system, and $S_{1}, S_{2}, S_{3}, S_{4}$ are the independence systems for $A_{1}, A_{2}, A_{3}, A_{4}$ respectively. The systems are all Boolean.

\begin{tabular}{|c|c|c|}
\hline \hline System & $K$ & $a \supset b$ \\
\hline$S_{0}$ & 0,1 & $a^{\prime}+b$ \\
$S_{1}$ & 0,1 & $0 / 0^{*}$ \\
$S_{2}$ & 0,1 & 0 \\
$S_{3}$ & 0,1 & $a$ \\
$S_{4}$ & 0 & 0 \\
\hline
\end{tabular}

4. Theorems. The proof of the sufficiency of Postulates $A_{1}-A_{4}$ for Boolean algebra, and for the theory of deduction of the Principia, will be effected with the help of the following theorems 1a-26a.

1a. $(a \supset z) \supset z=a$.

2a. $(a \supset a) \supset b=b$.

3a. $(a \supset b) \supset c=\{[(c \supset z) \supset a] \supset[(b \supset c) \supset z]\}>z$.

4a. $(a \supset z) \supset b=(b \supset z) \supset a$.

$5 a$.

$$
a \supset b=(b \supset z) \supset(a \supset z) \text {. }
$$

6a. $a \supset(b \supset z)=b \supset(a \supset z)$.

7a. The element $z$ of Postulate $\mathrm{A}_{3}$ is unique.

Definition 1a. $a_{1}=a \supset z$.

8 .

9a.

10a.

11a.

12a.

13a.

$14 a$.

$$
\begin{gathered}
a_{11}=a, \text { where } a_{11}=\left(a_{1}\right)_{1} . \\
(a \supset b) \supset c=\left[\left(c_{1} \supset a\right) \supset(b \supset c)_{1}\right]_{1} .
\end{gathered}
$$

$$
a_{1} \supset b=b_{1} \supset a \text {. }
$$$$
a \supset b=b_{1} \supset a_{1} \text {. }
$$$$
a \supset b_{1}=b \supset a_{1} \text {. }
$$

$$
a_{1}=a \supset a_{1} \text {. }
$$

$$
a \supset a=b \supset b \text {. }
$$

* In a two-element Boolean algebra, we may define the quotient precisely as in the case of the algebra of number. 
Definition 2a. $u=a \supset a$.

$15 a$.

$16 a$.

$17 \mathrm{a}$.

$18 \mathrm{a}$.

19a.

20a.

21a.

$22 a$.

23a.

$24 a$.

$$
z \supset a=u \text {. }
$$$$
u \supset a=a \text {. }
$$

$$
z_{1}=u ; \quad u_{1}=z .
$$$$
a \supset b=\left(\begin{array}{lll}
b & a_{1}
\end{array}\right) \supset a_{1} \text {. }
$$$$
(a \supset b) \supset b=b_{1} \supset a \text {. }
$$

$$
a \supset b=b_{1} \supset(a \supset b) \text {. }
$$$$
a \supset b=a \supset(a \supset b) \text {. }
$$$$
a \supset b=(b \supset a) \supset(a \supset b) \text {. }
$$

Definition 3a. $a \mid b=b \supset a_{1}$.

Definition 4a. $a^{\prime}=a \mid a$.

25a.

$$
a^{\prime}=a_{1}
$$

DeFINITION 5a. $\sim a=a_{1}$.

DEFINITION 6a. $a \vee b=a_{1} \supset b$.

26a.

$$
\sim a \vee b=a \supset b .
$$

Definition 7a. $\vdash a=(a=u)$.

5. Proofs of the theorems. The proofs of the theorems 1a-26a follow.

Proof of 1a. $a=(a \supset a) \supset a=(a \supset a) \supset[(a \supset z) \supset a]=\{[(a \supset z) \supset a] \supset$ $[(z \supset a) \supset z]\} \supset z=(a \supset z) \supset z$, by $\mathrm{A}_{2}, \mathrm{~A}_{2}, \mathrm{~A}_{3}, \mathrm{~A}_{2}$.

Proof of 2a. $(a \supset a) \supset b=(a \supset a) \supset[(b>z)>b]=\{[(b>z)>b] \supset[(z \supset b)$ $\supset z]\} \supset z=(b \supset z) \supset z=b$, by $A_{2}, A_{3}, A_{2}, 1 \mathrm{a}$.

Proof of 3a. $(a \supset b) \supset c=(d \supset d) \supset[(a \supset b) \supset c]=\{[(c \supset z)>a] \supset[(b \supset c) \supset$ $z$ ] \} $\supset z$, by $2 a, A_{3}$.

Proof of 4a. $(a \supset z) \supset b=\{[(b \supset z) \supset a] \supset[(z \supset b) \supset z]\} \supset z=\{[(b \supset z) \supset a]$ $\supset z\} \supset z=(b \supset z) \supset a$, by $3 \mathrm{a}, \mathrm{A}_{2}, 1 \mathrm{a}$.

Proof of 5a. $a \supset b=[(a \supset z) \supset z] \supset b=(b \supset z) \supset(a \supset z)$, by 1a, 4a.

Proof of 6a. $a \supset(b \supset z)=[(b \supset z) \supset z] \supset(a \supset z)=b \supset(a \supset z)$, by 5a, 1a.

Proof of 7a. Suppose that two elements, $y$ and $z$, have the property of $z$. Then $y=(y \supset z) \supset z=\{[(z \supset y) \supset y] \supset[(z \supset z) \supset y]\} \supset y=\{z \supset[(z \supset z) \supset y]\} \supset y$ $=(z \supset y) \supset y=z$, by $1 \mathrm{a}, 3 \mathrm{a}, 1 \mathrm{a}, 2 \mathrm{a}, 1 \mathrm{a}$.

Proof of 8a. By def. 1a, 1a.

Proof of 9a. By def. 1a, 3a.

Proof of 10a. By def. 1a, 4a. 
Proof of 11a. By def. 1a, 5a.

Proof of 12a. By def. 1a, 6a.

Proof of 13a. $a_{1}=\left(a_{1} \supset z\right) \supset a_{1}=[(a \supset z) \supset z] \supset a_{1}=a \supset a_{1}$, by $A_{2}$, def. 1a, 1a.

Proof of 14a. $a \supset a=[(a \supset a) \supset z] \supset z=z \supset z=[(b \supset b) \supset z] \supset z=b \supset b$, by 1a, 2a, 2a, 1ay

Proof of 15a. $z \supset a=[(z \supset a) \supset z] \supset z=z \supset z=u$, by 1a, $\mathrm{A}_{2}$, def. 2a.

Proof of 16a. $u \supset a=(a \supset a) \supset a=a$, by def. $2 \mathrm{a}, \mathrm{A}_{2}$.

Proof of 17a. $z_{1}=z \supset z=u$, by def. 1a, def. 2a; $u_{1}=(z \supset z)_{1}=(z \supset z) \supset z=z$, by def. 2a, def. 1a, $A_{2}$.

Proof of 18a. $\left(b \supset a_{1}\right) \supset a_{1}=\left[\left(a_{11} \supset b\right) \supset\left(a_{1} \supset a_{1}\right)_{1}\right]_{1}=\left[\left(a_{1} \supset a_{1}\right) \supset\left(a_{11} \supset b\right)_{1}\right]_{1}=$ $\left(a_{11} \supset b\right)_{11}=a \supset b$, by $9 \mathrm{a}, 12 \mathrm{a}, 2 \mathrm{a}, 8 \mathrm{a}$.

Proof of 19a. $b_{1} \supset a=\left(a \supset b_{11}\right) \supset b_{11}=(a \supset b) \supset b$, by 18a, 8a.

Proof of 20a. $b_{1} \supset(a \supset b)=[(a \supset b) \supset b] \supset b=\left(b_{1} \supset a\right) \supset b=\left(a_{1} \supset b\right) \supset b=$ $b_{1} \supset a_{1}=a \supset b$, by $19 \mathrm{a}, 19 \mathrm{a}, 10 \mathrm{a}, 19 \mathrm{a}, 11 \mathrm{a}$.

Proof of 21a. $a \supset(a \supset b)=\left[(a \supset b) \supset a_{1}\right] \supset a_{1}=\left[\left(a_{11} \supset a\right) \supset\left(b \supset a_{1}\right)_{1}\right]_{1} \supset a_{1}=$ $\left[(a \supset a) \supset\left(b \supset a_{1}\right)_{1}\right]_{1} \supset a_{1}=\left(b \supset a_{1}\right)_{11} \supset a_{1}=\left(b \supset a_{1}\right) \supset a_{1}=a \supset b$, by 18a, 9a, 8a, 2a, 8a, 18a.

Proof of 22a. $(b \supset a) \supset(a \supset b)=\left\{\left[(a \supset b)_{1} \supset b\right] \supset[a \supset(a \supset b)]_{1}\right\}_{1}=\left\{\left[(a \supset b)_{1}\right.\right.$ $\left.\supset b] \supset(a \supset b)_{1}\right\}_{1}=(a \supset b)_{11}=a \supset b$, by $9 \mathrm{a}, 21 \mathrm{a}, \mathrm{A}_{2}, 8 \mathrm{a}$.

Proof of 23a. $a \supset(b \supset a)=\left[(b \supset a) \supset a_{1}\right] \supset a_{1}=\left[\left(a_{11} \supset b\right) \supset\left(a \supset a_{1}\right)_{1}\right]_{1} \supset a_{1}=$ $\left[\left(a_{11} \supset b\right) \supset a_{11}\right]_{1} \supset a_{1}=[(a \supset b) \supset a]_{1} \supset a_{1}=a_{1} \supset a_{1}=u$, by 18a, 9a, 13a, 8a, A, def. $2 a$.

Proof of 24a. $(b \supset a) \supset(b \supset c)=\left\{\left[(b \supset c)_{1} \supset b\right] \supset[a \supset(b \supset c)]_{1}\right\}_{1}=\left\{\left[b_{1} \supset\right.\right.$ $\left.(b \supset c)] \supset[a \supset(b \supset c)]_{1}\right\}_{1}=\left\{\left[b_{1} \supset\left(c_{1} \supset b_{1}\right)\right] \supset[a \supset(b \supset c)]_{1}\right\}_{1}=\{u \supset[a \supset(b \supset$ c) $\left.]_{1}\right\}_{1}=[a \supset(b \supset c)]_{11}=a \supset(b \supset c)$, by $9 \mathrm{a}, 10 \mathrm{a}, 11 \mathrm{a}, 23 \mathrm{a}, 16 \mathrm{a}, 8 \mathrm{a}$.

Proof of 25a. $a^{\prime}=a \mid a=a \supset a_{1}=a_{1}$, by def. $4 \mathrm{a}$, def. $3 \mathrm{a}, 13 \mathrm{a}$.

Proof of 26a. $\sim a \vee b=a_{1} \vee b=a_{11} \supset b=a \supset b$, by def. 5a, def. 6a, $8 \mathrm{a}$.

6. Sufficiency of the postulates. I shall now prove the sufficiency of postulates $\mathrm{A}_{1}-\mathrm{A}_{4}$ by deriving from them my second set of postulates for Boolean algebra in terms of rejection.* This set has as primitive ideas $K$ and "|," and as postulates the propositions $\mathbf{B}_{1}-\mathbf{B}_{4}$ following (in postulates $\mathbf{B}_{3}$ and $\mathbf{B}_{4}$ there is to be understood the supposition that the elements involved and their indicated combinations belong to $K$ ).

$B_{1}$. $K$ contains at least two distinct elements.

$\mathrm{B}_{2}$. If $a$ and $b$ are elements of $K, a \mid b$ is an element of $K$.

DEFINITION 1b. $a^{\prime}=a \mid a$.

$\mathrm{B}_{3}$.

$$
\begin{aligned}
a & =(b \mid a) \mid\left(b^{\prime} \mid a\right) . \\
a \mid(b \mid c) & =\left[\left(c^{\prime} \mid a\right) \mid\left(b^{\prime} \mid a\right)\right]^{\prime} .
\end{aligned}
$$

$\mathrm{B}_{4}$.

* See my Paper II, loc. cit. 
The derivations of $B_{1}-B_{4}$ from $A_{1}-A_{4}$ follow.

Proof of $B_{1}$. By $A_{4}$.

Proof of $B_{2}$. By def. 3a, def. 1a, $A_{1}$.

Proof of $\mathrm{B}_{3}$. $a=\left(a_{1}\right)_{1}=\left[(b \supset b) \supset a_{1}\right]_{1}=\left[\left(a_{11} \supset b\right) \supset\left(b \supset a_{1}\right)_{1}\right]_{11}=(a \supset b) \supset$ $\left(b \supset a_{1}\right)_{1}=\left(b \supset a_{1}\right) \supset(a \supset b)_{1}=\left(b \supset a_{1}\right) \supset\left(b_{1} \supset a_{1}\right)_{1}=(b \mid a)\left|\left(b_{1} \mid a\right)=(b \mid a)\right|\left(b^{\prime} \mid a\right)$, by $8 \mathrm{a}, 2 \mathrm{a}, 9 \mathrm{a}, 8 \mathrm{a}, 12 \mathrm{a}, 11 \mathrm{a}$, def. 3a, 25a.

Proof of $\mathrm{B}_{4} \cdot a \mid(b \mid c)=a \supset\left(b \supset c_{1}\right)_{1}=\left(b \supset c_{1}\right) \supset a_{1}=\left[\left(a_{11} \supset b\right) \supset\left(c_{1} \supset a_{1}\right)_{1}\right]_{1}=$ $\left[\left(b_{1} \supset a_{1}\right) \supset\left(c_{1} \supset a_{1}\right)_{1}\right]_{1}=\left[\left(c_{1} \supset a_{1}\right) \supset\left(b_{1} \supset a_{1}\right)_{1}\right]_{1}=\left[\left(c^{\prime} \supset a_{1}\right) \supset\left(b^{\prime} \supset a_{1}\right)_{1}\right]^{\prime}=\left[\left(c^{\prime} \mid a\right)\right.$ $\left.\mid\left(b^{\prime} \mid a\right)\right]^{\prime}$, by def. 3a, 12a, 9a, 10a, 12a, 25a, def. 3a.

7. Necessariness of the postulates. I shall prove that $A_{1}-A_{4}$ are necessary for Boolean algebra by deriving $A_{1}-A_{4}$ from the rejection postulates $B_{1}-B_{4}$ above. For this derivation $I$ shall use as auxiliary theorems propositions $1 \mathrm{~b}-8 \mathrm{~b}$ following, derivable from $\mathrm{B}_{1}-\mathrm{B}_{4}$.

$1 \mathrm{~b}$. $a^{\prime \prime}=a$, where $a^{\prime \prime}=\left(a^{\prime}\right)^{\prime}$.

$2 \mathrm{~b}$.

$$
a|b=b| a \text {. }
$$

$3 b$.

$$
a \mid\left(b \mid b^{\prime}\right)=a^{\prime} .
$$

$4 b$.

$$
(a \mid c) \mid(b \mid c)=\left[c \mid\left(a^{\prime} \mid b^{\prime}\right)\right]^{\prime} . .
$$

$5 \mathrm{~b}$.

$$
a\left|a^{\prime}=b\right| b^{\prime} \text {. }
$$

Definition 2b. $u=a \mid a^{\prime}$.

$6 \mathrm{~b}$.

$$
\begin{aligned}
a \mid u & =a^{\prime} . \\
a \mid u^{\prime} & =u .
\end{aligned}
$$

$7 \mathrm{~b}$.

Definition 3b. $a \supset b=a \mid b^{\prime}$.

$8 \mathrm{~b}$.

$$
a \supset u^{\prime}=a^{\prime} .
$$

Propositions $1 \mathrm{~b}, 2 \mathrm{~b}, 3 \mathrm{~b}, 5 \mathrm{~b}$ are respectively Sheffer's Postulate 3 , Theorem A, Postulate 4, Theorem B.* The proofs of $4 \mathrm{~b}, 6 \mathrm{~b}, 7 \mathrm{~b}$, and $8 \mathrm{~b}$ follow.

Proof of $4 \mathrm{~b} .\left[c \mid\left(a^{\prime} \mid b^{\prime}\right)\right]^{\prime}=\left[\left(b^{\prime \prime} \mid c\right) \mid\left(a^{\prime \prime} \mid c\right)\right]^{\prime \prime}=(b \mid c) \mid(a \mid c)$, by $\mathrm{B}_{4}, 1 \mathrm{~b}$.

Proof of 6b. $a|u=a|\left(a \mid a^{\prime}\right)=a^{\prime}$, by def. 2b, 3b.

Proof of $7 \mathrm{~b} . \quad a \mid u^{\prime}=\left[\left(a \mid u^{\prime}\right)^{\prime}\right]^{\prime}=\left[\left(a \mid u^{\prime}\right) \mid\left(a \mid a^{\prime}\right)\right]^{\prime}=\left[\left(u^{\prime} \mid a\right) \mid\left(a^{\prime} \mid a\right)\right]^{\prime}=$ $\left[a \mid\left(u^{\prime \prime} \mid a^{\prime \prime}\right)\right]^{\prime \prime}=a|(u \mid a)=a|(a \mid u)=a \mid a^{\prime}=u$, by $1 \mathrm{~b}, 3 \mathrm{~b}, 2 \mathrm{~b}, 4 \mathrm{~b}, 1 \mathrm{~b}, 2 \mathrm{~b}, 6 \mathrm{~b}$, def. $2 \mathrm{~b}$.

Proof of $8 \mathrm{~b} . a \supset u^{\prime}=a\left|u^{\prime \prime}=a\right| u=a^{\prime}$, by def. $3 \mathrm{~b}, 1 \mathrm{~b}, 6 \mathrm{~b}$.

The derivations of $A_{1}-A_{4}$ from $B_{1}-B_{4}$ now follow.

Proof of $A_{1}$. By def. $3 b$, def. $1 b, B_{2}$.

Proof of $A_{2} .(a \supset b) \supset a=\left(a \mid b^{\prime}\right)\left|a^{\prime}=\left(a \mid b^{\prime}\right)\right|\left[a \mid\left(b \mid b^{\prime}\right)\right]=\left(b^{\prime} \mid a\right) \mid\left[\left(b^{\prime} \mid b\right) \mid a\right]$

* See H. M. Sheffer, $A$ set of five independent postulates for Boolean algebras, with application to logical constants, these Transactions, vol. 14 (1913), pp. 481-488. 
$=\left\{a \mid\left[b^{\prime \prime} \mid\left(b^{\prime} \mid b\right)^{\prime}\right]\right\}^{\prime}=\left\{a \mid\left[b \mid\left(b^{\prime} \mid b\right)^{\prime}\right]\right\}^{\prime}=\left\{a \mid\left[b \mid\left(b \mid b^{\prime}\right)^{\prime}\right]\right\}^{\prime}=\left[a \mid\left(b \mid u^{\prime}\right)\right]^{\prime}=$ $(a \mid u)^{\prime}=a^{\prime \prime}=a$, by def. $3 \mathrm{~b}, 3 \mathrm{~b}, 2 \mathrm{~b}, 4 \mathrm{~b}, 1 \mathrm{~b}, 2 \mathrm{~b}$, def. $2 \mathrm{~b}, 7 \mathrm{~b}, 6 \mathrm{~b}, 1 \mathrm{~b}$.

Proof of $A_{3}$. The element $u^{\prime}$ will serve as the required element $z$. For, $\left\{\left[\left(c \supset u^{\prime}\right) \supset a\right] \supset\left[(b \supset c) \supset u^{\prime}\right]\right\} \supset u^{\prime}=\left[\left(c^{\prime} \supset a\right) \supset(b \supset c)^{\prime}\right]^{\prime}=\left[\left(c^{\prime} \mid a^{\prime}\right) \mid\left(b \mid c^{\prime}\right)^{\prime \prime}\right]^{\prime}$ $=\left[\left(c^{\prime} \mid a^{\prime}\right) \mid\left(b \mid c^{\prime}\right)\right]^{\prime}=\left[\left(a^{\prime} \mid c^{\prime}\right) \mid\left(b \mid c^{\prime}\right)\right]^{\prime}=\left\{\left[c^{\prime} \mid\left(a^{\prime \prime} \mid b^{\prime}\right)\right]^{\prime}\right\}^{\prime}=\left[c^{\prime} \mid\left(a^{\prime \prime} \mid b^{\prime}\right)\right]^{\prime}$ $\left|\left(d \mid d^{\prime}\right)=\left[c^{\prime} \mid\left(a \mid b^{\prime}\right)\right]^{\prime}\right|\left(d \mid d^{\prime}\right)=\left(d \mid d^{\prime}\right) \mid\left[\left(a \mid b^{\prime}\right) \mid c^{\prime}\right]^{\prime}=(d \supset d)>[(a \supset b) \supset c]$, by $8 b$, def. 3b, 1b, 2b, 4b, 3b, 1b, 2b, def. 3b.

Proof of $\mathrm{A}_{4}$. By $\mathrm{B}_{1}$.

8. Derivation of the theory of deduction. I now come to the derivation from $\mathrm{A}_{1}-\mathrm{A}_{4}$ of the theory of deduction of Principia Mathematica. The primitive ideas of this theory are a class $K$, a unary operation ", ," a binary operation " $v$," and a notion "ト," which may perhaps be termed a predicative relation. The postulates of the theory are the propositions $\mathrm{C}_{1}-\mathrm{C}_{7}$ below. These postulates are expressed in terms of $K, \sim, \vee, \vdash$, and an operation " $\supset$ " defined by

Definition 1c. $a \supset b=\sim a \vee b$.

By 26a, the " $\supset$ " of Definition 1c is seen to be the same as the " $\supset$ " of postulates $A_{1}-A_{4}$. This fact will be used hereafter without further mention. The postulates $\mathrm{C}_{1}-\mathrm{C}_{7}$ follow. $\dagger$

$\mathrm{C}_{1}\left[{ }^{*} 1 \cdot 1\right]$. If $\vdash a$ and $\vdash(a \supset b)$ then $\vdash b$.

$\mathrm{C}_{2}\left[*_{1} \cdot 2\right] . \vdash[(a \vee a)>a]$.

$\mathrm{C}_{3}\left[*_{1} \cdot 3\right] . \vdash[a \supset(b \vee a)]$.

$\mathrm{C}_{4}\left[{ }^{*} 1 \cdot 4\right] . \vdash[(a \vee b) \supset(b \vee a)]$.

$\mathrm{C}_{5}\left[*_{1} \cdot 6\right] . \vdash\{(a \supset b) \supset[(c \vee a) \supset(c \vee b)]\}$.

$\mathrm{C}_{6}\left[{ }^{*} 1 \cdot 7\right]$. If $a$ is in $K$, then $\sim a$ is in $K$.

$\mathrm{C}_{7}\left[{ }^{*} 1 \cdot 71\right]$. If $a$ and $b$ are in $K$, then $a \vee b$ is in $K$.

The derivations of $\mathrm{C}_{1}-\mathrm{C}_{7}$ from $\mathrm{A}_{1}-\mathrm{A}_{4}$ follow.

Proof of $\mathrm{C}_{1}$. Let $\vdash a$ and $\vdash(a \supset b)$. Then $a=u$ and $a \supset b=u$, by def. $7 \mathrm{a}$. Hence $u \supset b=u$. Hence $b=u$, by $16 \mathrm{a}$; hence $\vdash b$, by def. $7 \mathrm{a}$.

Proof of $\mathrm{C}_{2}$. $(a \vee a) \supset a=\left(a_{1} \supset a\right) \supset a=a_{1} \supset a_{1}=u$, by def. 6a, 19a, def. 2a. Hence the theorem, by def. 7a.

Proof of $\mathrm{C}_{3} . a \supset(b \vee a)=a \supset\left(b_{1} \supset a\right)=u$, by def. 6a, 23a. Hence the theorem, by def. 7a.

Proof of $\mathrm{C}_{4} .(a \vee b) \supset(b \vee a)=\left(a_{1} \supset b\right) \supset\left(b_{1} \supset a\right)=\left(a_{1} \supset b\right) \supset\left(a_{1} \supset b\right)=u$, by def. 6a, 10a, def. 2a. Hence the theorem, by def. 7a.

Proof of $C_{5}$. $(a \supset b) \supset[(c \vee a) \supset(c \vee b)]=(a \supset b) \supset\left[\left(c_{1} \supset a\right) \supset\left(c_{1} \supset b\right)\right]=$

† The numbers associated with $\mathrm{C}_{1}-\mathrm{C}_{7}$ are those of the Principia. For the form of $* 1 \cdot 1$, see my (VII) Remarks on propositions *1.1 and *3.35 of Principia Mathematica, Bulletin of the American Mathematical Society, vol. 39 (1933), pp. 111-114. The Principia proposition *1 5 has been omitted, since ${ }^{*} 1.5$ is redundant (see P. Bernays, Mathematische Zeitschrift, vol. 25 (1926), pp. 305-320). 
$(a \supset b) \supset\left[a \supset\left(c_{1} \supset b\right)\right]=b \supset\left[a \supset\left(c_{1} \supset b\right)\right]=\left\{\left[a \supset\left(c_{1} \supset b\right)\right] \supset b_{1}\right\} \supset b_{1}=\left\{\left(b_{11} \supset a\right)\right.$ $\left.\supset\left[\left(c_{1} \supset b\right) \supset b_{1}\right]_{1}\right\}_{1} \supset b_{1}=\left\{(b \supset a) \supset\left[\left(c_{1} \supset b\right) \supset b_{1}\right]_{1}\right\}_{1} \supset b_{1}=\{(b \supset a)$ $\left.\supset\left[\left(b_{1} \supset c\right) \supset b_{1}\right]_{1}\right\}_{1} \supset b_{1}=\left[(b \supset a) \supset b_{11}\right]_{1} \supset b_{1}=[(b \supset a) \supset b]_{1} \supset b_{1}=b_{1} \supset b_{1}$ $=u$, by def. 6a, 24a, 24a, 18a, 9a, 8a, 10a, $\mathrm{A}_{2}, 8 \mathrm{a}, \mathrm{A}_{2}$, def. $2 \mathrm{a}$. Hence the theorem, by def. $7 \mathrm{a}$.

Proof of $\mathrm{C}_{6}$. By def. 5a, def. 1a, $\mathrm{A}_{1}$.

Proof of $\mathrm{C}_{7}$. By def. 6a, def. 1a, $\mathrm{A}_{\mathrm{i}}$.

9. Relation between the implicative operation and the operation exception. I shall now bring out the relation existing between the implicative operation $\supset$ and the operation "

The considerations are simple. The element $a-b$ is, in the usual Boolean notation, the element $a b^{\prime}$. Since $a \supset b$ is the element $a^{\prime}+b$, the elements $a \supset b$ and $b-a$ are the duals of each other. Hence, $a$ postulate-set in terms of $\supset$ is essentially also a set in terms of " -," and vice versa.

Let me actually transform Postulates $\mathrm{A}_{1}-\mathrm{A}_{4}$ into a set in terms of "-." To do this, it will be convenient to re-letter the formulas in $A_{1}-A_{4}$. If we write $b-a$ for $a \supset b, z$ for $u$ (the dual of $z$ ), and re-letter, Postulates $A_{1}-A_{4}$ become the following postulates $\mathrm{D}_{1}-\mathrm{D}_{4}$ in terms of "exception."

$D_{1} . a-b$ is an element of $K$ whenever $a$ and $b$ are elements of $K$.

$\mathrm{D}_{2} \cdot a-(b-a)=a$.

$\mathrm{D}_{\mathbf{3}}$. There is an element $u$ in $K$ such that

$$
[a-(b-c)]-(d-d)=u-\{[u-(a-b)]-[c-(u-a)]\} \text {. }
$$

$\mathrm{D}_{4} . K$ consists of at least two distinct elements.

To actually transform a set of "exception" postulates into a set of "implication" postulates, let me take a set due to Taylor.* This set consists of the postulates $\mathrm{E}_{1}-\mathrm{E}_{5}$ following. $\dagger$

$\mathrm{E}_{1} . K$ contains at least two distinct elements.

$\mathrm{E}_{2}$. If $a$ and $b$ are elements of $K, a-b$ is an element of $K$.

$\mathrm{E}_{3} . a-(b-b)=a$.

$\mathrm{E}_{4}$. There exists a unique element $u$ in $K$ such that $a-(u-b)=b-(u-a)$.

Definition 1e. $a_{1}=u-a$.

$\mathrm{E}_{5} . a-(b-c)=\left[(a-b)_{1}-\left(a-c_{1}\right)\right]_{1}$.

If we replace $a-b$ by $b \supset a$ and $u$ by $z$, and re-letter, $\mathrm{E}_{1}-\mathrm{E}_{5}$ become the following postulates $F_{1}-F_{5}$ in terms of implication.

$F_{1} . K$ contains at least two distinct elements.

$\mathrm{F}_{2}$. If $a$ and $b$ are elements of $K, a \supset b$ is an element of $K$.

F. $(a \supset a) \supset b=b$.

* J. S. Taylor, loc. cit.

$\dagger$ In $E_{3}, E_{4}, E_{6}$ there is to be understood the supposition that the elements involved and their indicated combinations belong to $K$. In $\mathrm{E}_{8}$ there is to be understood the further supposition that $\mathrm{E}_{4}$ holds. 
$\mathrm{F}_{4}$. There exists a unique element $z$ such that $(a \supset z) \supset b=(b \supset z) \supset a$.

Definition 1f. $a_{1}=a \supset z$.

F $_{5} .(a \supset b) \supset c=\left[\left(a_{1} \supset c\right) \supset(b \supset c)_{1}\right]_{1}$.

10. Postulates for the logic of propositions. I take up finally the last item of my paper: to show that a simple change in one of the postulates $\mathrm{A}_{1}-\mathrm{A}_{4}$ will make these postulates a set of $i n d e p e n d e n t$ postulates for the logic of propositions as a two-element Boolean algebra. The change consists in replacing Postulate $\mathrm{A}_{4}$ by Postulate $\mathrm{A}_{4}^{\prime}$ following:

Postulate $\mathrm{A}_{4}^{\prime} . K$ consists of two distinct elements.

That $\mathbf{A}_{1}, \mathbf{A}_{2}, \mathbf{A}_{3}, \mathbf{A}_{4}^{\prime}$ are necessary and sufficient for a two-element Boolean algebra, is obvious. That $\mathrm{A}_{1}, \mathrm{~A}_{2}, \mathrm{~A}_{3}, \mathrm{~A}_{4}^{\prime}$ are mutually independent, is seen from the table of $\S 3$ : in that table each of the independence systems for $A_{1}, A_{2}$, $\mathrm{A}_{3}$ consists of only two elements.*

* In the same way, and for the same reasons, one can transform into independent postulatesets for the logic of propositions my two sets for Boolean algebra in terms of rejection (see my Papers I, II, loc. cit.). A like remark applies to Huntington's first set of postulates for Boolean algebra (E. V. Huntington, (III) Sets of independent postulates for the algebra of logic, these Transactions, vol. 5 (1904), pp. 288-309).

University of California, Berkeley, Calif. 